\title{
The young woman who could not stop vomiting
}

\author{
A P de Silva, A Molagoda, P L N Fernando, H J de Silva
}

An unmarried, unemployed, 28-year-old woman presented to us with self-induced vomiting, reduced sleep, and crying spells. Four years ago, she had begun thinking that she and her family were obese. She started taking only liquids and induced vomiting. She used bees honey and lemon juice in the belief that these would reduce her weight. One year later she developed spontaneous vomiting. She lost weight rapidly and developed amenorrhoea a few months later. She was third in a family of seven. The family system was one of parental dominance, with no emotional support. On examination, she was depressed and emaciated, with parotid enlargement, bradycardia and fine lanugo hair. Other systems were clinically normal. A barium meal was done (figure). The following investigations were also performed: haemoglobin $7 \mathrm{~g} / \mathrm{dl}$, prolactin $52 \mathrm{ng} / \mathrm{ml}$ (normal 0.33-27.3), follicle-stimulating hormone (FSH) $1.57 \mathrm{IU} / 1$ (18-153), luteinising hormone (LH) $0.45 \mathrm{IU} / 1$ (16-24), potassium $3 \mathrm{mmol} / \mathrm{l}$, sodium $125 \mathrm{mmol} / \mathrm{l}$. Serum cholesterol, serum proteins, creatinine, erythrocyte sedimentation rate, urine examination, and chest $\mathrm{X}$-ray were normal. Subsequently, a surgical procedure was performed. She recovered from surgery but after initial improvement, she started inducing vomiting again, and refused food. There was no response to prokinetic drugs.

Faculty of Medicine, University of Kelaniya, P O Box 6, Thalagolla Road, Ragama, Sri

Lanka

Department of

Medicine

A P de Silva

H J de Silva

Department of

Psychiatry

A Molagoda

Psychiatry Unit, Colombo North

General Hospital,

Ragama, Sri Lanka

P L N Fernando

Accepted 23 April 1998

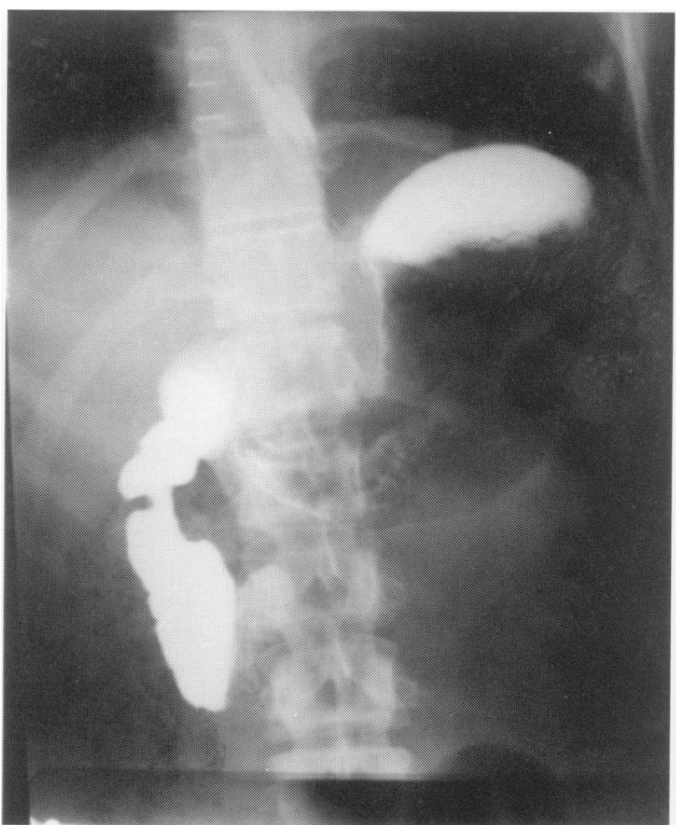

\section{Questions}

1 What does the barium meal show?

2 What additional manoeuvre could be done at the time of performing the barium meal to confirm the radiological diagnosis?

3 What was the most probable diagnosis at surgery?

4 What was the underlying cause?

Figure Barium meal 


\section{Answers}

QUESTION 1

The barium meal shows obstruction to the third part of the duodenum with proximal dilation. There is a sharp cut off of the barium column.

QUESTION 2

The Hayes manoeuvre, in which there is disappearance of the obstruction in the knee-chest position on cinefluoroscopy, would confirm the radiological diagnosis.

\section{QUESTION 3}

Superior mesenteric artery syndrome (SMAS), a condition in which there is obstruction to the third part of the duodenum by the superior mesenteric artery against the underling aorta. The predisposing factors are an acute aortomesenteric vascular angle, exaggerated lumbar lordosis and abnormally high fixation of the duodenojejunal flexure to the ligament of Treitz. The main precipitating cause is marked weight loss which results in loss of mesenteric and retroperitoneal fat, thus causing an abnormally acute aortomesenteric angle. ${ }^{1}$

\section{QUESTION 4}

In this patient chronic SMAS was due to anorexia nervosa. Anorexia nervosa consists of three core features, altered body image and size, loss of weight more than $15 \%$ of the normal, and amenorrhoea. ${ }^{2}$ In addition, increased prolactin with decreased FSH and LH are characteristic findings. Electrolyte imbalance may occur due to prolonged vomiting. All these features were present in our patient. Gastrointestinal disorders are common in anorexia nervosa. SMAS is a rare but recognised complication of the disease. ${ }^{3}$

The principles of treatment in anorexia nervosa are two-fold. First, establish healthy eating habits and a normal weight, which is achieved by gradual re-feeding. Second, remove those factors which have been maintaining the disorder, which involve the use of family therapy and cognitive behaviour therapy. ${ }^{4}$

\section{Discussion}

Although anorexia nervosa is classically a disease of adolescent girls from western countries and upper social class, it can occur in any ethnic group or social class. ${ }^{5}$ The delay of two years in making the diagnosis in this patient emphasises the fact that anorexia nervosa should always be considered in young women with gastrointestinal disorders associated with vomiting and marked weight loss.

\section{Final diagnosis}

Superior mesenteric artery syndrome due to anorexia nervosa.

Keywords: superior mesenteric artery syndrome; anorexia nervosa
1 Ahmed AR, Taylor I. Superior mesenteric artery syndrome. Postgrad Med F 1997;73:777-8.

2 Elabadaway MH. Chronic superior mesenteric artery syndrome in anorexia nervosa. Br $\mathcal{F}$ Psychiatry 1995;160: 552-4.

3 Pentlow BD. Acute vascular compression of the duodenum in anorexia nervosa. Br $\mathcal{F}$ Surg 1981;68:665-6.
4 Hartman D. Anorexia nervosa- diagnosis, aetiology and treatment. Postgrad Med f 1995;71:712-6.

5 Wichstrom I. Social psychological and physical correlates of eating problems, a study of general adolescent population in Norway. Psychol Med 1995;25:567-79. 